\title{
A Genome-Wide Survey of Genes for Enzymes Involved in Pigment Synthesis in an Ascidian, Ciona intestinalis
}

\author{
Keisuke Takeuchi ${ }^{1}$, Yutaka Satou ${ }^{1}$, Hiroaki Yamamoto $^{2}$ and Nori Satoh ${ }^{1 *}$ \\ ${ }^{1}$ Department of Zoology, Graduate School of Science, Kyoto University, Kyoto 606-8502, Japan \\ ${ }^{2}$ Department of Developmental Biology and Neurosciences, Graduate School of \\ Life Sciences, Tohoku University, Sendai, Miyagi 980-8578, Japan
}

\begin{abstract}
The draft genome sequence and a large quantity of EST and CDNA information are now available for the ascidian Ciona intestinalis. In the present study, genes involved in pigment synthesis pathways were identified in the decoded genome of Ciona, and information about these genes was obtained from available EST and cDNA sequences. It was found that the Ciona genome contains orthologous genes for each enzyme of the melanin, pteridine, ommochrome, papiliochrome, and heme synthesis pathways. Several appear as independent duplications in the Ciona genome. Because cDNA clones for all but two of these genes have already been isolated by the CDNA project, $C$. intestinalis will provide an experimental system to explore molecular mechanisms underlying color patterns, through future genome-wide studies.
\end{abstract}

Key words: Ciona intestinalis, pigment synthesis, genes, genome-wide survey

\section{INTRODUCTION}

Animals exhibit various colors and color patternings. The color pattern is not only specific to certain species of animals but also is altered by season to season, day to day, or in response to environmental cues. The color patterning is accomplished by a complex combination of pigments. In zebrafish, for example, black melanophores, yellow xanthophores and iridescent iridophores are involved in the pigment pattern formation (Quigley and Parichy, 2002). Animal pigments are formed through the melanin synthesis pathway, pteridine synthesis pathway, ommochrome synthesis pathway, and papiliochrome synthesis pathway.

Ascidians are marine invertebrate chordates, comprising approximately 2,300 species, and they exhibit various colors and patterns. For example, Clavelina species in tropical sea are very bright blue, and Halocynthia roretzi adults are red. Ciona intestinalis and Ciona savignyi are closely related species, and the presence or absence of red pigment spots in the rim of the sperm duct is used as one of the diagnostic characters to distinguish between the two species (Hoshino and Tokioka, 1967). We are interested in how various color patterns are achieved in ascidians, and in particular, molecular mechanisms underlying the difference in pigment pattern between the two Ciona species. So far, genes for enzymes required for melanin pigment forma-

\footnotetext{
* Corresponding author. Phone: $+81-75-753-4081$; Fax : +81-75-705-1113; E-mail: satoh@ascidian.zool.kyoto-u.ac.jp
}

tion have been studied in only one ascidian, Halocynthia roretzi (Sato et al., 1997; Sato et al., 1999). In order to understand color patterns in ascidians in a genome-wide sense, genes associated with pigment synthesis pathways should be completely annotated. The aim of the present study was to describe how many and what kinds of relevant genes, with or without redundancy, are encoded in Ciona intestinalis, whose genome was recently decoded (Dehal et al., 2002).

\section{MATERIALS AND METHODS}

Retrieving sequences from the Ciona intestinalis genome and a cDNA/EST database

All of the methods used in the present study were as described by Satou et al. (2003a). The draft genome sequence (Dehal et al., 2002) and a cDNA/EST database (Satou et al., 2002) of Ciona intestinalis were TBlastN searched for homologous ascidian protein sequences using human and Drosophila proteins. At least one human enzyme protein and all of the known Drosophila enzyme proteins involved in each pigment synthetic pathway were used for the search. When the corresponding cDNA sequence was available, the deduced protein sequence was used for the analyses. When the cDNA sequence was not available and GrailEXP or Genewise confidently predicted the gene, the peptide sequence deduced from the gene model was used (the gene model names are listed in Table 1). When the predicted gene model was not perfect but the ESTs covered the entire region or the region the gene model lacked, we used the peptide sequence deduced from the assembled sequences of ESTs, multiple sets of ESTs, or from both an EST and the gene model. 
Table 1. Genes for enzymes involved in pigment synthesis in the Ciona intestinalis genome

\begin{tabular}{|c|c|c|c|c|c|c|}
\hline Pathway & Abbreviations & Gene name & $\begin{array}{l}\text { The best gene model } \\
\text { in the version } 4 \text { assembly }\end{array}$ & $\begin{array}{l}\text { cDNA } \\
\text { cluster }\end{array}$ & $\begin{array}{l}\text { Best hit } \\
\text { analysis }^{b}\end{array}$ & $\begin{array}{l}\text { Other supporting } \\
\text { evidence }\end{array}$ \\
\hline \multirow[t]{5}{*}{ melanin synthesis } & TYR & tyrosinase & grail.166.38.1 & 14634 & $\rightarrow$ & \\
\hline & TYRP 1/2-a & tyrosinase-related protein $1 / 2-a$ & grail.42.3.1 & 32248 & $\leftrightarrow$ & \\
\hline & TYRP 1/2-b & tyrosinase-related protein $1 / 2-b$ & grail.13.78.1 & 14021 & $\rightarrow$ & \\
\hline & PO-like-a & phenoloxidase-like-a & CAD68059 (Scaffold 176) & 07503 & $\leftrightarrow \mathrm{c}$ & \\
\hline & PO-like-b & phenoloxidase-like-b & CAD68058 (Scaffold 12) & 11746 & $\rightarrow^{c}$ & \\
\hline \multirow[t]{10}{*}{ pteridine synthesis } & $\mathrm{GCHI}$ & GTP cyclohydrolase I & grail.884.2.1 & 05238 & $\leftrightarrow$ & \\
\hline & PTPS & 6-pyruvoyl H4pterin synthase & grail.6.149.1 & 11056 & $\leftrightarrow$ & \\
\hline & SPR-a & sepiapterin reductase-a & grail.62.76.1 & 03905 & $\leftrightarrow$ & \\
\hline & SPR-b & sepiapterin reductase-b & genewise.116.150.1 & 15808 & $\rightarrow$ & \\
\hline & $\mathrm{XO} / \mathrm{XDH}-\mathrm{a}$ & xanthine oxidase/xanthine dehydrogenase-a & grail.613.1.1 & 06608 & $\leftrightarrow$ & domain composition \\
\hline & $\mathrm{XO} / \mathrm{XDH}-\mathrm{b}$ & xanthine oxidase/xanthine dehydrogenase-b & genewise8.31.1 & 14640 & $\rightarrow$ & domain composition \\
\hline & clot-a & clot-a & grail.665.4.1 & 13855 & $\leftrightarrow$ & CXXC motif \\
\hline & clot-b & clot-b & genewise.239.39.1 & $N A^{a}$ & $\rightarrow$ & CXXC motif \\
\hline & $\mathrm{PCD} / \mathrm{DcoH}$ & $\begin{array}{l}\text { pterin } 4 a-c a r b i n o l a m i n e \\
\text { dehydratase/dimerization cofactor of } \\
\text { hepatocyte nuclear factor } 1\end{array}$ & genewise.30.367.1 & 03895 & $\leftrightarrow$ & \\
\hline & DHPR & dihydropteridine reductase & genewise.11.378.1 & 05796 & $\leftrightarrow$ & \\
\hline \multirow[t]{4}{*}{ ommochrome synthesis } & TDO2-a & tryptophan 2,3-dioxygenase-a & grail.124.18.1 & 13900 & $\rightarrow$ & \\
\hline & TDO2-b & tryptophan 2,3-dioxygenase-b & grail.1428.1.1 & 16081 & $\leftrightarrow$ & \\
\hline & KF-like & kynurenine formamidase-like & genewise.103.226.1 & 07539 & $\rightarrow$ & $\begin{array}{l}\text { HGG motif, GXSXG } \\
\text { motif }\end{array}$ \\
\hline & $\mathrm{KMO}$ & kynurenine 3-monooxygenase & grail.171.37.1 & 03910 & $\leftrightarrow$ & \\
\hline \multirow[t]{2}{*}{ papiliochrome synthesis } & DDC/HDC-like-a & dopa decarboxylase-like-a & genewise.412.21.1 & NA & $\rightarrow$ & \\
\hline & DDC/HDC-like-b & dopa decarboxylase-like-b & genewise.324.101.1 & 16852 & $\leftrightarrow$ & \\
\hline \multirow[t]{8}{*}{ heme synthesis } & ALAS & $\delta$-aminolevulinate synthase & grail.42.37.1 & 09795 & $\leftrightarrow$ & \\
\hline & ALAD & $\delta$-aminolevulinate dehydratase & grail.145.14.1 & 31091 & $\leftrightarrow$ & \\
\hline & PBGD & porphobilinogen deaminase & grail.758.5.1 & 08121 & $\leftrightarrow$ & \\
\hline & UPG III S & uroporphyrinogen III synthase & genewise.103.196.1 & 04049 & $\leftrightarrow$ & \\
\hline & UPD & uroporphyrinogen decarboxylase & grail.755.6.1 & 06538 & $\leftrightarrow$ & \\
\hline & $\mathrm{CPO}$ & coproporphyrinogen oxidase & grail.126.52.1 & 01962 & $\rightarrow$ & \\
\hline & PPO & protoporphyrinogen oxidase & grail.268.12.1 & 36273 & $\leftrightarrow$ & \\
\hline & ferrochelatase & ferrochelatase & grail.726.2.1 & 04286 & $\leftrightarrow$ & \\
\hline
\end{tabular}

\footnotetext{
a NA, not available

b “ $\leftrightarrow$ ” indicates a bi-directional best-hit relationship between a Ciona gene and a human protein, and “ $\rightarrow$ " indicates a uni-directional best-hit relationship of a Ciona protein against a human protein.

c The result was obtained using the Drosophila melanogaster proteome, because the human proteome did not appear to contain the most likely protein.
}

\section{Motif search}

Motifs or domains of retrieved protein sequences were examined using SMART (Schultz et al., 1998) and the PFAM database (Bateman et al., 2002).

\section{Molecular phylogenetic analysis}

Sequences were aligned using the CLUSTAL program (Higgins and Sharp, 1988) and the alignment was checked by eye. After removal of gaps, the verified alignments were used to construct phylogenetic trees. Trees were calculated with the MEGA program using the neighbor-joining method (Saitou and Nei, 1987; Kumar et al., 2001). Maximum-likelihood analyses were also performed when needed, using the Phylip 3.6 package (Felsenstein, 1993). Sequences included are represented by accession number, abbreviation of species (see below), and gene name. For example, human cPKC-b (accession number P05771) is represented as "P05771 HS cPKC-b". All sequences used in the present study are available on request. Abbreviations of species are HS for Homo sapiens, MM for
Mus musculus, RN for Rattus norvegicus, GG for Gallus gallus, XL for Xenopus laevis, TN for Tetraodon nigroviridis, TR for Takifugu rubripes, DR for Danio rerio, HR for Halocynthia roretzi, BF for Branchiostoma floridae, CE for Caenorhabditis elegans, DM for Drosophila melanogaster, AG for Anopheles gambiae, AT for Arabidopsis thaliana, PC for Petroselinum crispum, PS for Papaver somniferum, SC for Saccharomyces cerevisiae, SP for Schizosaccharomyces pombe, EC for Escherichia coli, DD for Dictyostelium discoideum, BC for Bacillus cereus, BA for Bacillus anthracis, HP for Helicobacter pylori J99, LM for Leishmania major, DR for Deinococcus radiodurans, and ML for Mesorhizobium loti. Abbreviations of species shown in Fig. 2 are PL for Pacifastacus leniusculus (crustacean), PS for Penaeus semisulcatus (crustacean), MJ for Marsupenaeus japonicus (crustacean), PV for Penaeus vannamei (crustacean), PM for Penaeus monodon (crustacean), PI for Panulirus interruptus (crustacean), PL for Pontastacus leptodactylus (crustacean), SP for Spirostreptus sp. BT-2000 (myriapod), SC for Scutigera coleoptrata (myriapod), NI for Nephila inaurata madagas- 
cariensis (myriapod), CS for Cupiennius salei (myriapod), and EP for Epiperipatus sp. TB-2001 (onychophoran).

\section{Best-hit analysis}

To confirm the results of the molecular phylogenetic analyses, we compared the indicated Ciona proteins with the human and Drosophila SWISS-PROT/TrEMBL proteome sets released on 17 and 24 Aug. 2002, respectively. Identified proteins were first compared using the BlastP program (Altschul et al., 1997). The best-hit protein in each proteome was then TBlastN searched against the Ciona genome without the option of gapped alignment. When the best-hit sequence of the human or Drosophila protein corresponded to the region encoding the starting Ciona protein, the relationship between the two proteins was called the "bi-directional best-hit relationship", and supported the orthology of the two proteins. Otherwise, it was called a "uni-directional best-hit relationship".

\section{EST counts}

A large scale EST analysis was conducted for transcripts expressed in Ciona intestinalis. The cDNA libraries examined were from fertilized eggs, cleaving embryos, gastrulae/neurulae, tailbud embryos, larvae and whole young adults, and the gonad (ovary and testis), endostyle, neural complex, heart, and blood cells of the adult. Because the libraries were not normalized or amplified, the occurrence of cDNA clones or EST counts in each library may reflect the quantity of transcripts of the corresponding genes. Thus, comparison of the EST counts of a certain gene at the six developmental stages listed above may reflect the temporal expression pattern of the gene, while the comparison of EST counts in different tissues of the adult may reflect the spatial expression pattern of the gene (Satou et al., 2003b).

\section{RESULTS AND DISCUSSION}

\section{Melanin synthesis pathways}

Melanin is found in both plants and animals, although its structural features differ between the two taxa. The present study dealt with only eumelanin, not allomelanin, which is found in plants, fungi and bacteria, nor pheomelanin, which is only found in birds and mammals. Fig. 1A shows the synthesis pathway of melanin and enzymes involved in the pathway (reviewed by Hearing and Tsukamoto, 1991; del Marmol and Beermann, 1996). The key enzymes are tyrosinase (TYR) and tyrosinase-related protein (TYRP). In mice, some mutations in the tyrosinase gene result in an albino phenotype that lacks melanin, and those in TYRP1 and/or TYRP2 alter coat color. Ascidian genes for TYR and TYRP have been isolated and characterized in Halocynthia roretzi (Sato et al., 1997; Sato et al., 1999).

The present survey of the Ciona intestinalis genome revealed the presence of a single gene for tyrosinase $(T Y R)$ and two genes (TYRP1/2-a and TYRP1/2-b) for TYRP (Fig. 1B; Table 1). The EST counts suggest that both TYR and TYRP are expressed in tailbud-stage embryos and larvae (Table 2). Since melanin is a component of the otolith and ocellus in the sensory vesicle of larvae, the gene expression is associated with the pigment formation.
A

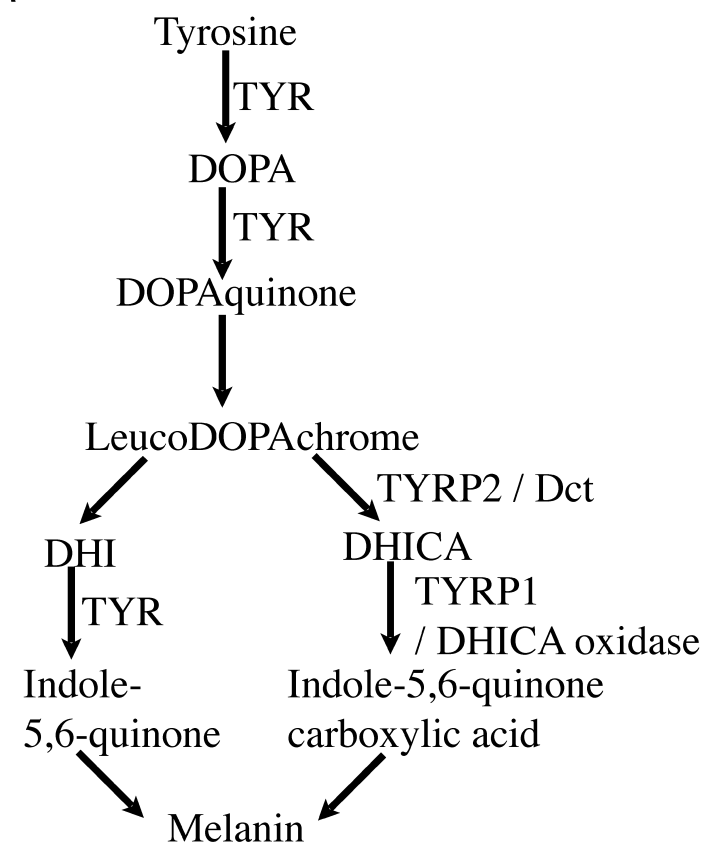

B

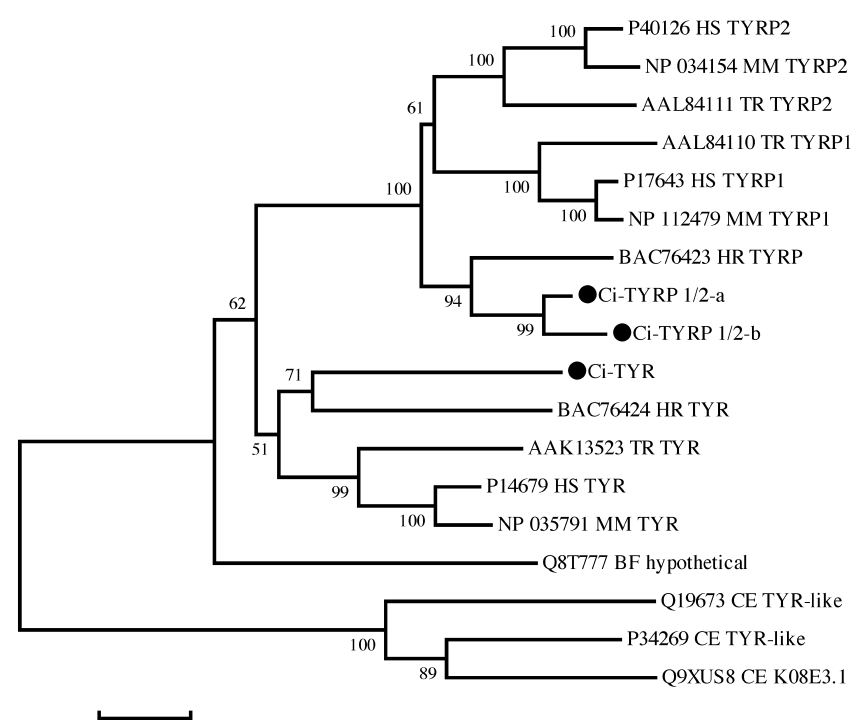

Fig. 1. (A) Melanin biosynthesis pathway based on description by Hearing and Tsukamoto (1991) and del Marmol and Beermann (1996). Dct, dopachrome tautomerase; DHI, 5,6-dihydroxyindole; DHICA, 5,6-dihydroxyindole-2-carboxylic acid; DOPA, 3,4-dihydroxyphenylalanine; TYR, tyrosinase; and TYRP, tyrosinase-related protein. (B) Phylogenetic tree of enzymes (TYR, TYRP1 and TYRP2) used for melanin synthesis, generated by the neighbor joining method. Ciona intestinalis proteins are shown by large black dots. The number beside each branch indicates the percentage of times that a node was supported in 1000 bootstrap pseudoreplications. Protein names are explained in the Methods section. The unrooted tree is shown as a rooted tree for simplicity. The scale bar indicates an evolutionary distance of 0.1 amino acid substitutions per position. 
Table 2. EST counts of genes of enzymes used for melanin synthesis in Ciona intestinalis

\begin{tabular}{lcccccc}
\hline \multicolumn{1}{c}{ Gene } & EG & CL & GN & TB & LV & cDNA cluster \\
\hline TYR & 0 & 0 & 0 & 7 & 4 & 14634 \\
TYRP 1/2-a & 0 & 0 & 2 & 0 & 7 & 32248 \\
TYRP 1/2-b & 0 & 0 & 0 & 3 & 0 & 14021 \\
\hline
\end{tabular}

EG, eggs; CL, cleaving embryos; GN, gastrulae and neurulae; TB, tailbud embryos; LV, larvae.

TYR, tyrosinase; TYRP, tyrosinase-related protein.

In vertebrates, TYRP1 and TYRP2 show different enzymatic activities. TYRP1 has the activity of dihydroxyindole carboxylic acid oxidase (DHICA oxidase), while TYRP2 has the activity of dopachrome tautomerase (Dct, EC 5.3.3.12) and is therefore generally called Dct. In addition, copper binds to the active site of tyrosinase, whereas zinc binds to that of TYRP2, but what binds to that of TYRP1 is still unknown (García-Borrón and Solano, 2002). Molecular phylogenetic analysis showed that TYRP1/2-a and $T Y R P 1 / 2-b$ resulted from an independent duplication in the lineage of this ascidian (Fig. 1B), suggesting that both $T Y R P 1 / 2-a$ and $T Y R P 1 / 2-b$ are an ancestral form of the two TYRPs of vertebrates, which were duplicated in the lineage leading to vertebrates. Therefore, the manner of melanin synthesis in Ciona is likely the ancestral form of that in vertebrates.

In the melanin synthesis pathway in insects, phenoloxidase (PO, EC 1.10.3.1) acts in place of tyrosinase, and dopachrome isomerase in place of TYRP2 (dopachrome tautomerase). Insect dopachrome isomerase catalyses the conversion of dopachrome to 5,6-dihydroxyindole. TYRP1 appears to be lacking in the insect genome (reviewed by Sugumaran, 2002). The Ciona intestinalis genome contains

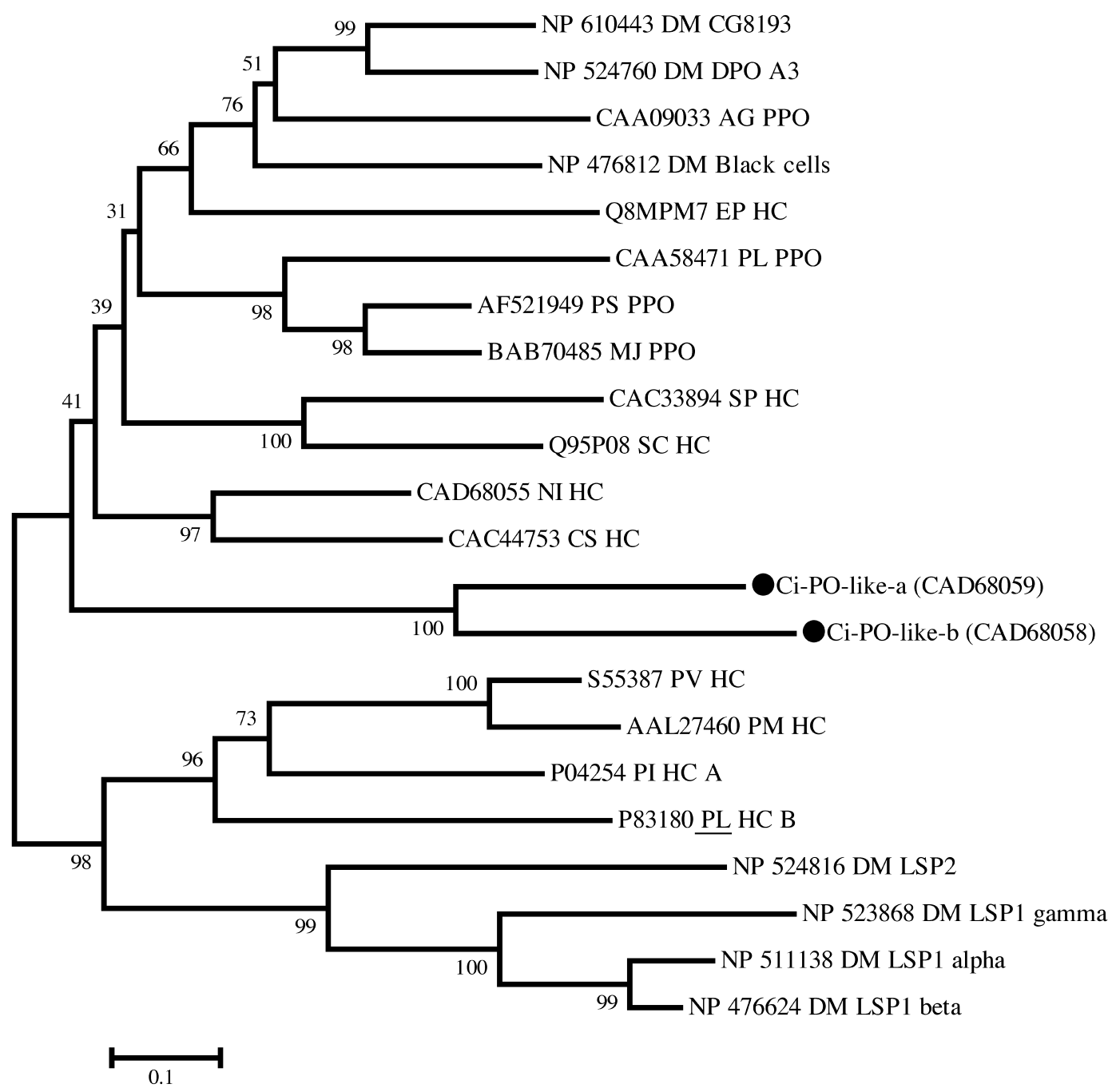

Fig. 2. Phylogenetic tree of phenoloxidase (PO) used for melanin synthesis, generated by the neighbor joining method. Ciona intestinalis proteins are shown by large black dots. The number beside each branch indicates the percentage of times that a node was supported in 1000 bootstrap pseudoreplications. Protein names are explained in the Methods section. The unrooted tree is shown as a rooted tree for simplicity. The scale bar indicates an evolutionary distance of 0.1 amino acid substitutions per position. DPO, diphenoloxidase; HC, hemocyanin; LSP, larval serum protein; and PPO, prophenoloxidase. 
two PO-like genes of the arthropod hemocyanin superfamily (Fig. 2) (Burmester, 2002; Immesberger and Burmester, 2004). On the other hand, the Ciona genome appears to lack a gene encoding the dopachrome conversion enzyme. As a result, although Ciona differs from vertebrates in that Ciona has PO-like genes, Ciona does not synthesize melanin in the same manner as insects.

\section{Pteridine synthesis pathway}

A well-known pteridine in plants and animals is $\mathrm{H}_{4}$ biopterin, which is utilized as a cofactor of enzymes. Pteridine is also used in pigmentation in animals including arthropods, teleost fishes, and amphibians. The pteridine synthesis pathway and enzymes involved in the pathway are shown in Fig. 3A (reviewed by Ziegler, 2003). This complex pathway is composed of three component pathways. The first is associated with the production of $\mathrm{H}_{4}$ biopterin from GTP, and involves three enzymes, GTP cyclohydrolase I (GCHI, EC 3.5.4.16), 6-pyruvoyl $\mathrm{H}_{4}$ pterin synthase (PTPS, EC 4.2.3.12), and sepiapterin reductase (SPR, EC 1.1.1.153). Mutations in mammalian genes encoding these enzymes result in the failure of $\mathrm{H}_{4}$ biopterin production, which causes various diseases such as hyperphenylalaninemia (reviewed by Thony et al., 2000). The second component pathway is the production of pigments such as drosopterin and sepiapterin from the middle products of the first pathway. The enzymes involved in this pathway are sepiapterin reductase, xanthine oxidase (XO, EC 1.1.3.22)/dehydrogenase (XDH, EC 1.1.1.204), and the clot gene product in Drosophila, whose mutations cause changes of eye color (Wiederrecht et al., 1984). The third component pathway is associated with regeneration after $\mathrm{H}_{4}$ biopterin acts as cofactor. Pterin 4a-carbinolamine dehydratase (PCD, EC 4.2.1.96) and dihydropteridine reductase (DHPR, EC 1.5.1.34) are
A

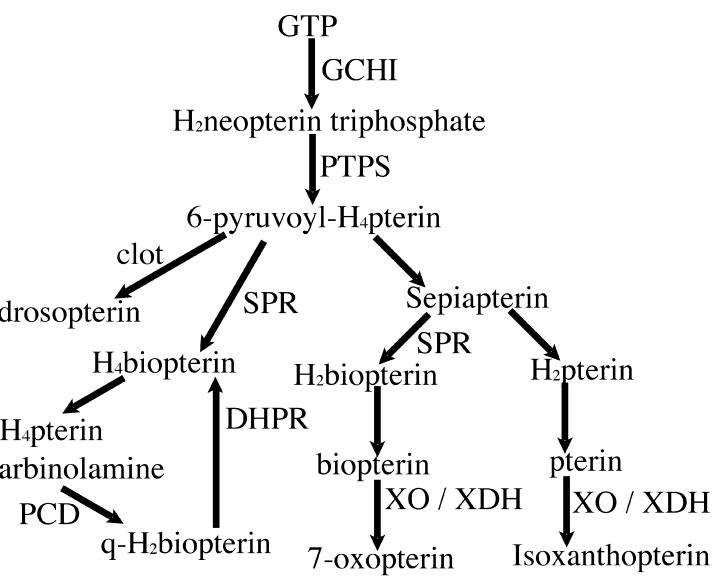

B

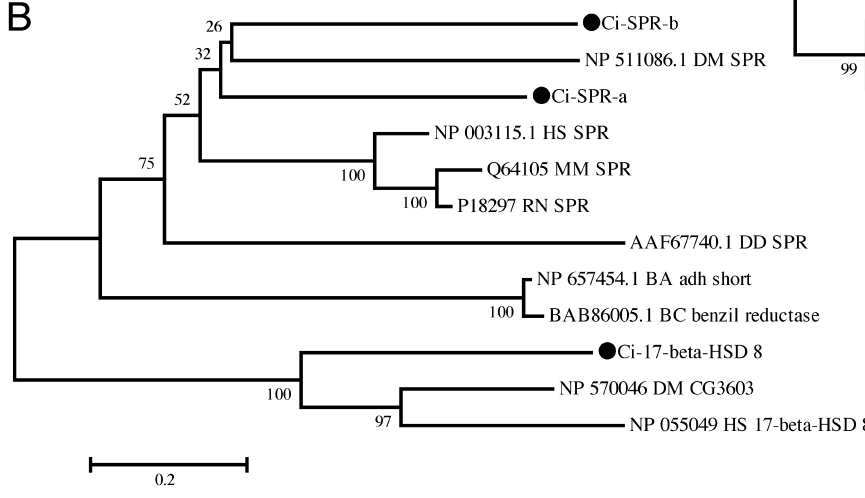

C

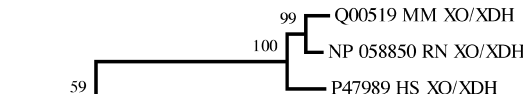

00 LP $058850 \mathrm{RN}$ XO/XDH

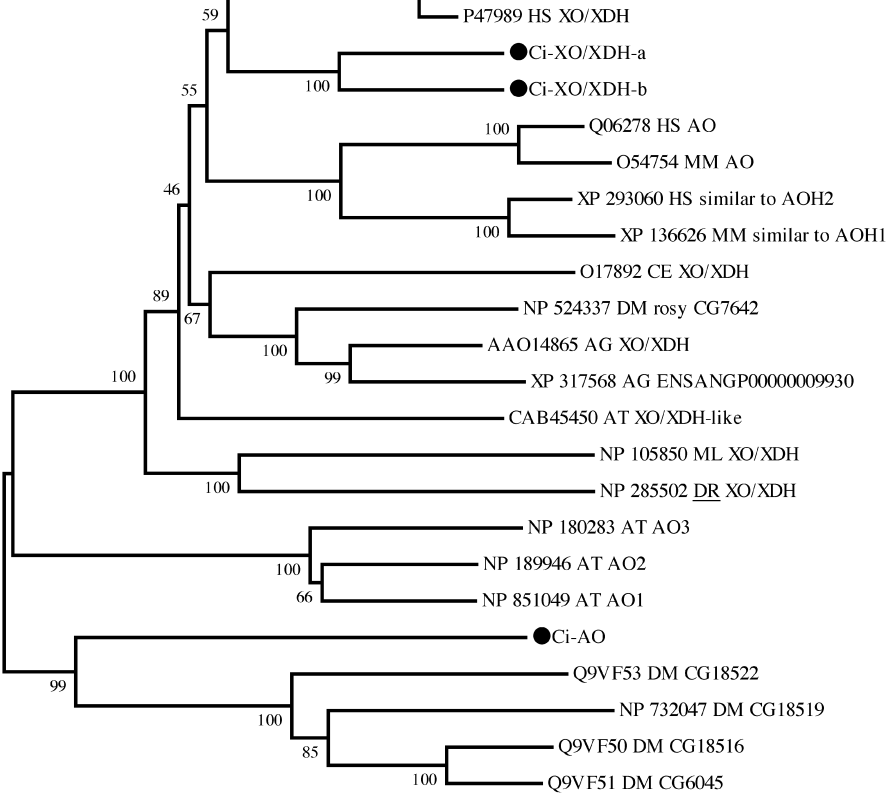

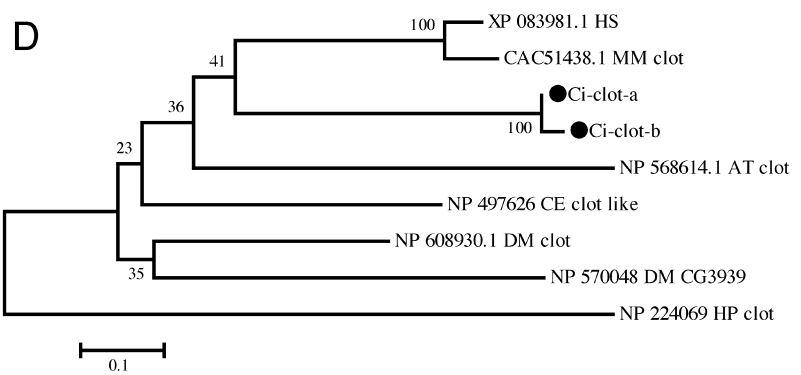

Fig. 3. (A) Pteridine biosynthesis pathway (Ziegler, 2003) and (B-D) phylogenetic tree of enzymes used for pteridine synthesis. The phylogenetic trees of $(B)$ sepiapterin reductase (SPR), (C) xanthine oxidase (XO)/xanthine dehydrogenase (XDH) and (D) clot were generated by the neighbor joining method. Ciona intestinalis proteins are shown by large black dots. The number beside each branch indicates the percentage of times that a node was supported in 1000 bootstrap pseudoreplications. Protein names are explained in the Methods section. Each unrooted tree is shown as a rooted tree for simplicity. The scale bar indicates an evolutionary distance of 0.2 (B) or 0.1 (C, D) amino acid substitutions per position. AO, aldehyde oxidase; DHPR, dihydropteridine reductase; GCHI, GTP cyclohydrolase I; 17-beta-HSD8, estradiol 17-beta-dehydrogenase 8; PCD, pterin 4a-carbinolamine dehydratase; and PTPS, 6-pyruvoyl- $\mathrm{H}_{4}$ pterin synthase. 

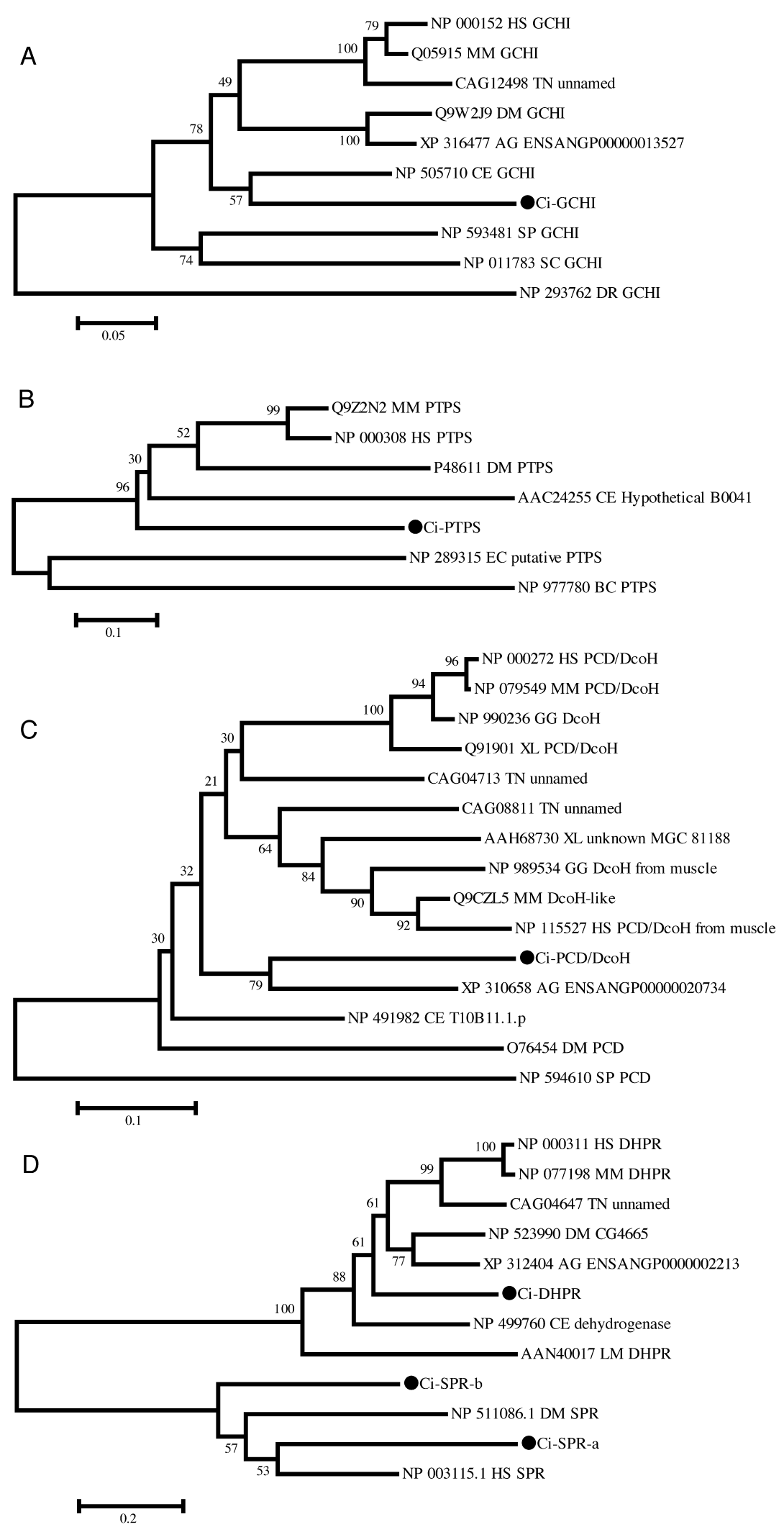

Fig. 4. Phylogenetic trees of enzymes used for pteridine synthesis. Phylogenetic trees of (A) GTP cyclohydrolase I (GCHI), (B) 6-pyruvoyl$\mathrm{H}_{4}$ pterin synthase (PTPS), (C) pterin 4a-carbinolamine dehydratase (PCD)/dimerization cofactor of hepatocyte nuclear factor 1(DcoH) and (D) dihydropteridine reductase (DHPR) were generated by the neighbor joining method. Ciona intestinalis proteins are shown by large black dots. The number beside each branch indicates the percentage of times that a node was supported in 1000 bootstrap pseudoreplications. Protein names are explained in the Methods section. Each unrooted tree is shown as a rooted tree for simplicity. The scale bar indicates an evolutionary distance of $0.05(A), 0.1(B, C)$ or $0.2(D)$ amino acid substitutions per position. SPR, sepiapterin reductase. 
Table 3. EST counts of genes of enzymes used for pteridine synthesis in Ciona intestinalis

\begin{tabular}{lccccccccccccc}
\hline Gene & EG & CL & GN & TB & LV & AD & GD & TS & ES & NC & HT & BD & cDNA cluster \\
\hline GCHI & 0 & 2 & 0 & 0 & 0 & 0 & 0 & 0 & 0 & 0 & 0 & 0 & 05238 \\
PTPS & 1 & 1 & 0 & 0 & 0 & 1 & 0 & 0 & 0 & 0 & 0 & 0 & 11056 \\
SPR-a & 0 & 2 & 0 & 0 & 0 & 1 & 1 & 0 & 0 & 0 & 0 & 0 & 03905 \\
SPR-b & 0 & 0 & 0 & 0 & 0 & 3 & 0 & 0 & 0 & 0 & 1 & 0 & 15808 \\
XO/XDH-a & 0 & 0 & 1 & 0 & 1 & 1 & 2 & 0 & 0 & 0 & 0 & 1 & 06608 \\
XO/XDH-b & 0 & 0 & 0 & 0 & 1 & 0 & 0 & 0 & 0 & 0 & 0 & 0 & 14640 \\
clot-a & 1 & 1 & 0 & 0 & 0 & 2 & 1 & 0 & 1 & 1 & 1 & 0 & 13855 \\
clot-b & 0 & 0 & 0 & 0 & 0 & 0 & 0 & 0 & 0 & 0 & 0 & 0 & not available \\
PCD/DcoH & 0 & 3 & 1 & 1 & 2 & 0 & 1 & 0 & 0 & 1 & 1 & 3 & 03895 \\
DHPR & 2 & 2 & 4 & 3 & 0 & 6 & 1 & 0 & 0 & 3 & 3 & 1 & 05796 \\
\hline
\end{tabular}

EG, eggs; CL, cleaving embryos; GN, gastrulae and neurulae; TB, tailbud embryos; LV, larvae; AD, Young adults; GD, gonad; TS, testis; ES, endostyle; NC, neural complex; HT, heart; BD, blood cells.

GCHI, GTP cyclohydrolase I; PTPS, 6-pyruvoyl- $H_{4}$ pterin synthase; SPR, sepiapterin reductase; XO, xanthine oxidase; $\mathrm{XDH}$, xanthine dehydrogenase; $\mathrm{PCD}$, pterin 4a-carbinolamine dehydratase; $\mathrm{DcoH}$, dimerization cofactor of hepatocyte nuclear factor 1; DHPR, dihydropteridine reductase.

A

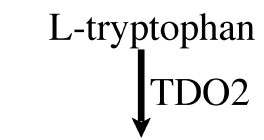

Formylkynurenine Kynurenin formamidase

L-Kynurenine

$\bigsqcup_{\text {3-Hydroxykynurenine }}^{\mid \text {KMO }}$
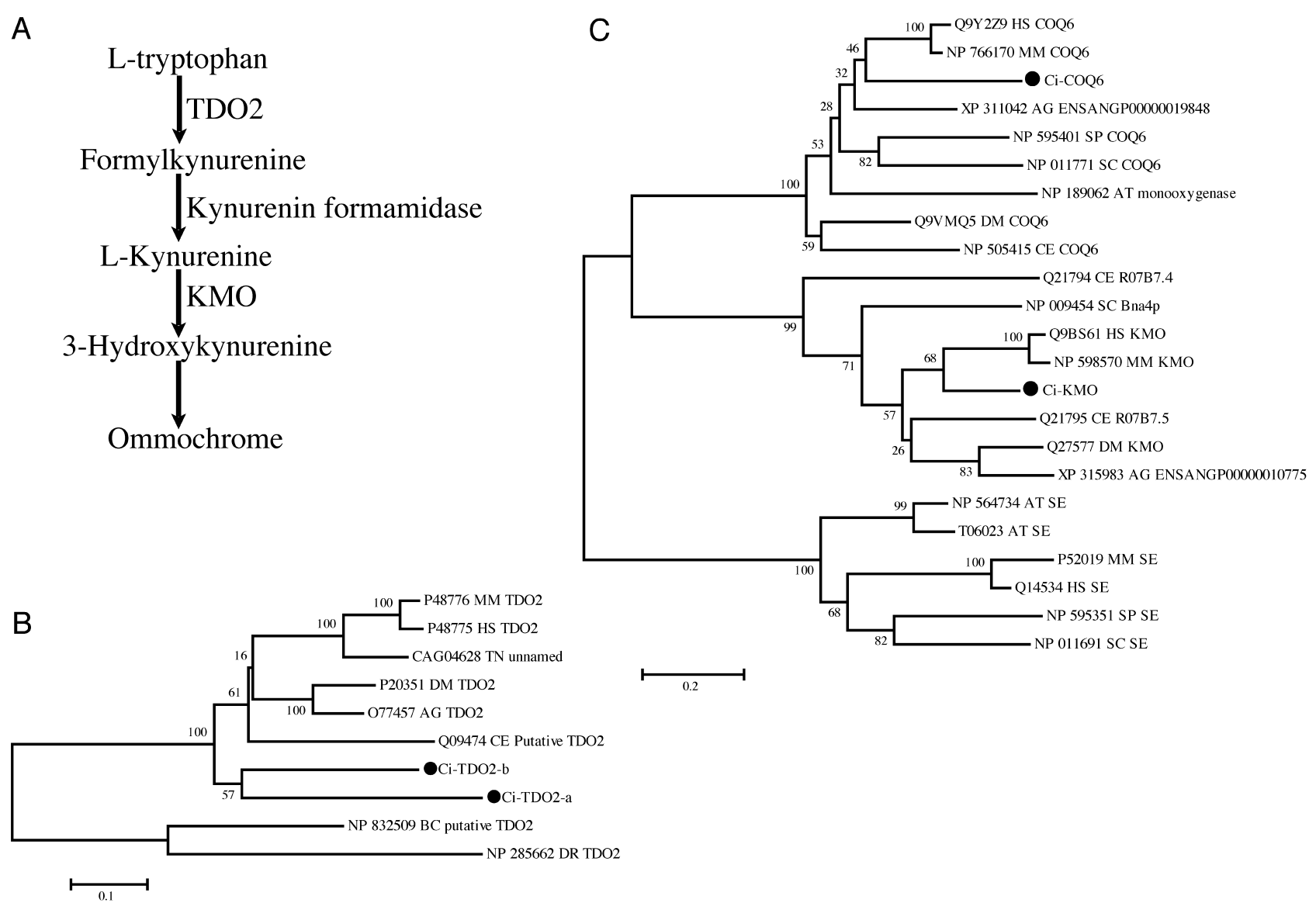

Fig. 5. (A) Ommochrome biosynthesis pathway (Han et al., 2003) and (B, C) phylogenetic tree of enzymes used for ommochrome synthesis. The phylogenetic tree of (B) tryptophan 2,3-dioxygenase (TDO2) and (C) kynurenine 3-monooxygenase (KMO) were generated by the neighbor joining method. Ciona intestinalis proteins are shown by large black dots. The number beside each branch indicates the percentage of times that a node was supported in 1000 bootstrap pseudoreplications. Protein names are explained in the Methods sections. Each unrooted tree is shown as a rooted tree for simplicity. The scale bar indicates an evolutionary distance of 0.1 (B) or 0.2 (C) amino acid substitutions per position. COQ6, Ubiquinone biosynthesis monooxygenase; and SE, squalene epoxidase. 
responsible for the pathway (Thony et al., 2000; Ziegler, 2003). Several mutations of the enzymes mentioned above cause changes in the pigmentation pattern of animals. For example, lemon is a mutant in the gene for SPR of the silkworm Bombyx mori (Matsubara et al., 1963). A Drosophila eye color mutant, rosy, and Bombyx skin color mutants, oq and og, occur in genes encoding xanthine dehydrogenase (Reaume et al., 1991; Tamura, 1983). In addition, three Drosophila eye-color mutants, purple, clot and sepia, are known (Wiederrecht et al., 1984). purple involves a mutation in the gene for 6-pyruvoyl $\mathrm{H}_{4}$ pterin synthase, and clot a mutation in the gene for thioredoxin-like protein (Giordano et al., 2003). sepia is thought to encode PDA synthase, but its nucleotide sequence has not fully been determined (Wiederrecht and Brown, 1984).

The search against the Ciona intestinalis genome revealed seven genes in the component pathways, each corresponding to the one of the enzymes mentioned above. In the first pathway, that of $\mathrm{H}_{4}$ biopterin biosynthesis, one gene for GTP cyclohydrolase I (GCHI) (Fig. 4A), one for 6pyruvoyl $\mathrm{H}_{4}$ pterin synthase (PTPS) (Fig. 4B), and two for sepiapterin reductase (SPR) (Fig. 3B) were detected (Table 1). The EST counts suggest that all these genes are expressed zygotically in embryos at the cleavage stage. In the second pathway, that for production of drosopterin, two genes for XO/XDH (Fig. 3C) and two clot homologs (Fig. 3D) were found in the Ciona genome. The EST counts suggested that these genes are expressed mainly in young adults after metamorphosis of tadpole larvae (Table 3). In the third pathway, that for regeneration of $\mathrm{H}_{4}$ biopterin, one gene for pterin 4a-carbinolamine dehydratase (PCD) (Fig. 4C) and one for dihydropteridine reductase (DHPR) (Fig. 4D) were found in the Ciona genome (Table 1). The Ciona PCD gene appeared to be an ancestral form of a gene that is duplicated in the vertebrate lineage (Fig. 4C). Ciona has two homologs each of SPR, XO/HDH, and clot in the genome. All of these genes appear to have been duplicated in the lineage of this ascidian, judging from the molecular phylogenetic analyses. Such lineage-specific duplications may be important for pigmentation in Ciona intestinalis, since all of them are involved in the second component pathway of pteridine synthesis. Therefore, it is highly likely that the pteridine synthesis path-
A

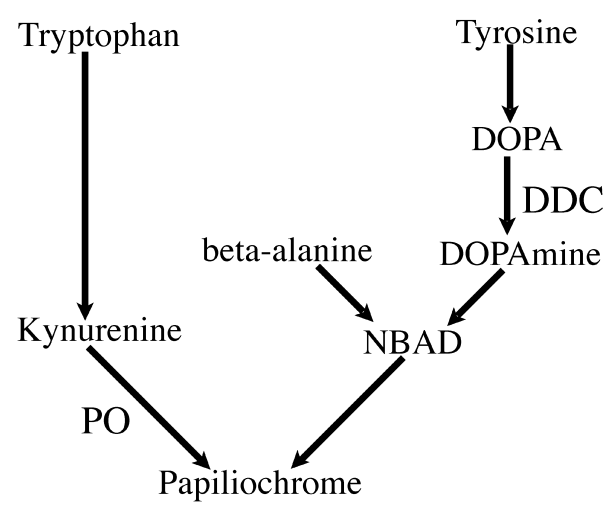

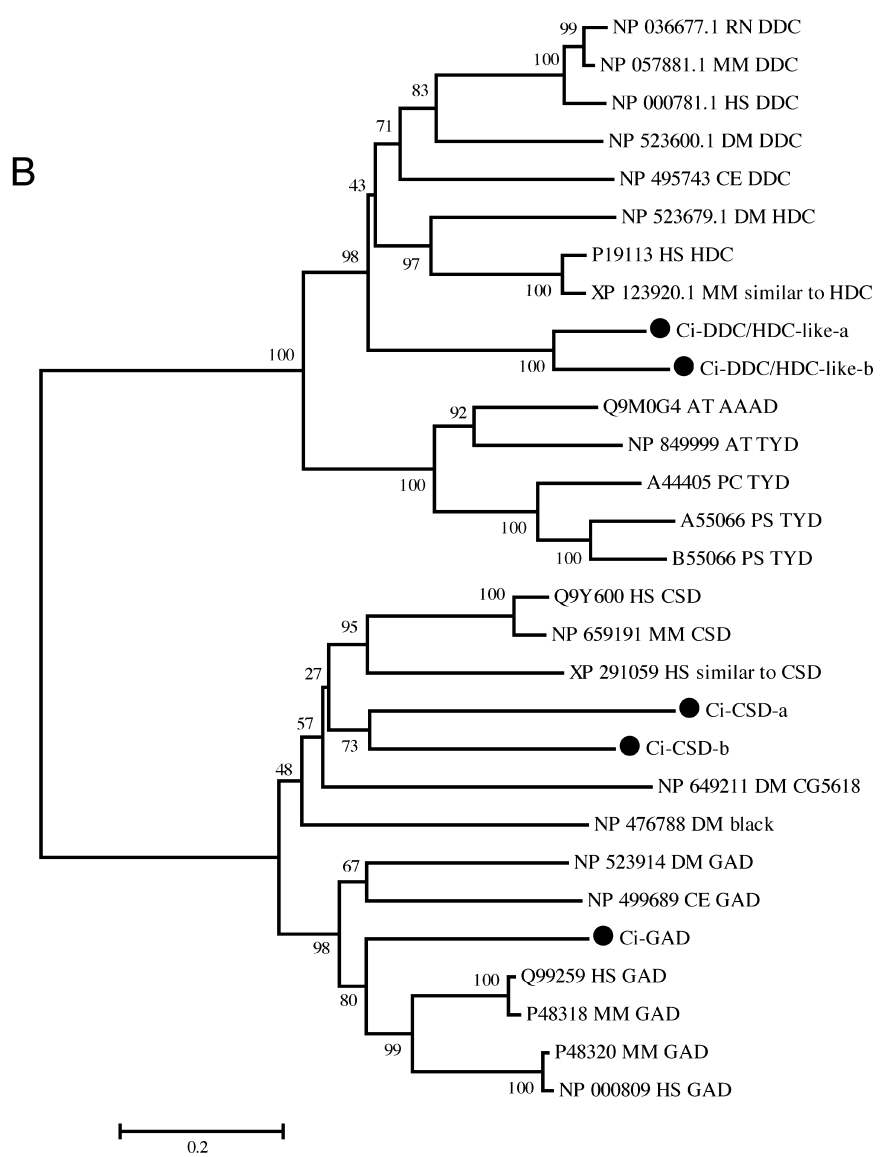

0.2

Fig. 6. (A) Papiliochrome biosynthesis pathway (Sugumaran et al., 1990; Koch et al., 2000) and (B) phylogenetic tree of enzymes used for papiliochrome synthesis. The phylogenetic tree of (B) dopa decarboxylase (DDC) was generated by the neighbor joining method. Ciona intestinalis proteins are shown by large black dots. The number beside each branch indicates the percentage of times that a node was supported in 1000 bootstrap pseudoreplications. Protein names are explained in the Methods section. The unrooted tree is shown as a rooted tree for simplicity. The scale bar indicates an evolutionary distance of 0.2 amino acid substitutions per position. AAAD, aromatic amino-acid decarboxylase; CSD, cysteine sulfinic acid decarboxylase; DOPA, 3,4-dihydroxyphenylalanine; GAD, galutamic acid decarboxylase; HDC, histidine decarboxylase; NBAD, N-beta-alanyldopamine; PO, phenoloxidase; and TYD, tyrosine decarboxylase. 
way is functional in Ciona intestinalis.

\section{Ommochrome synthesis pathway}

Ommochrome is well known as a pigment found in insect eyes, but it is also contained in eyes, hypostomes, wings, gonads, and ovaries of other arthropods, and molluscs as well. As shown in Fig. 5A, ommochrome is synthesized from 3-hydroxykynurenine, an intermediate product of the kynurenine pathway (Han et al., 2003). The kynurenine pathway involves tryptophan metabolism, and 3-hydroxykynurenine is synthesized from L-tryptophan. However, how ommochrome is synthesized from 3-hydroxykynurenine is not yet fully understood. Here we examined the kynurenine pathway, which involves three enzymes, tryptophan 2,3-dioxygenase (TDO2, EC 1.13.11.11), kynurenine formamidase (KF, EC 3.5.1.9), and kynurenine 3-monooxygenase (KMO, EC 1.14.13.9) (Allegri et al., 2003). In vertebrates, indoleamine 2,3-deoxygenase is used instead of TDO2, except in the liver. Although 3-hydroxykynurenine is usually used for biosynthesis other than pigment synthesis, some mutations that cause deficiency of 3-hydroxykynurenine change pigment patterns in insects, e.g., vermilion, a mutation in TDO2 of Drosophila (Searles and Voelker, 1986) and Tribolium (Lorenzen et al., 2002). Mutations in KMO are also known to cause abnormalities in eye pigmentation, e.g., cin- nabar in Drosophila (Warren et al., 1996), and similar mutations occur in Aedes aegypti (Han et al., 2003) and Tribolium (Lorenzen et al., 2002).

The present genomewide survey revealed two Ciona genes encoding tryptophan 2,3-dioxygenase (TDO2) (Fig. $5 \mathrm{~B}$ ) and one encoding kynurenine 3-monooxygenase (KMO) (Fig. 5C). The TDO2 genes appear to have been duplicated independently in the ascidian lineage. The mouse gene for kynurenine formamidase (KF) has been fully sequenced (Pabarcus and Casida, 2002), and the Ciona genome has a candidate gene for KF. A molecular phylogenetic analysis as well as structural features of the Ciona protein revealed that the GHSAG motif found in the mouse KF was replaced by GHSSG, and neither the catalytic unit nor the HGG motif was found in the protein. Therefore, we have termed this a KF-like gene. The two Ciona genes encoding TDO2 and the one encoding $\mathrm{KMO}$ are well conserved, suggesting that the kynurenine pathway functions in Ciona, but it is unclear whether this pathway is used for pigmentation in this genus.

\section{Papiliochrome synthesis pathway}

Papiliochrome is a yellow pigment characteristic of wings of butterflies belonging to the family Papilionidae. The synthesis pathway is shown in Fig. 6A. Papiliochrome synthesis requires N-beta-alanyldopamine (NBAD) and kynure-

A

B
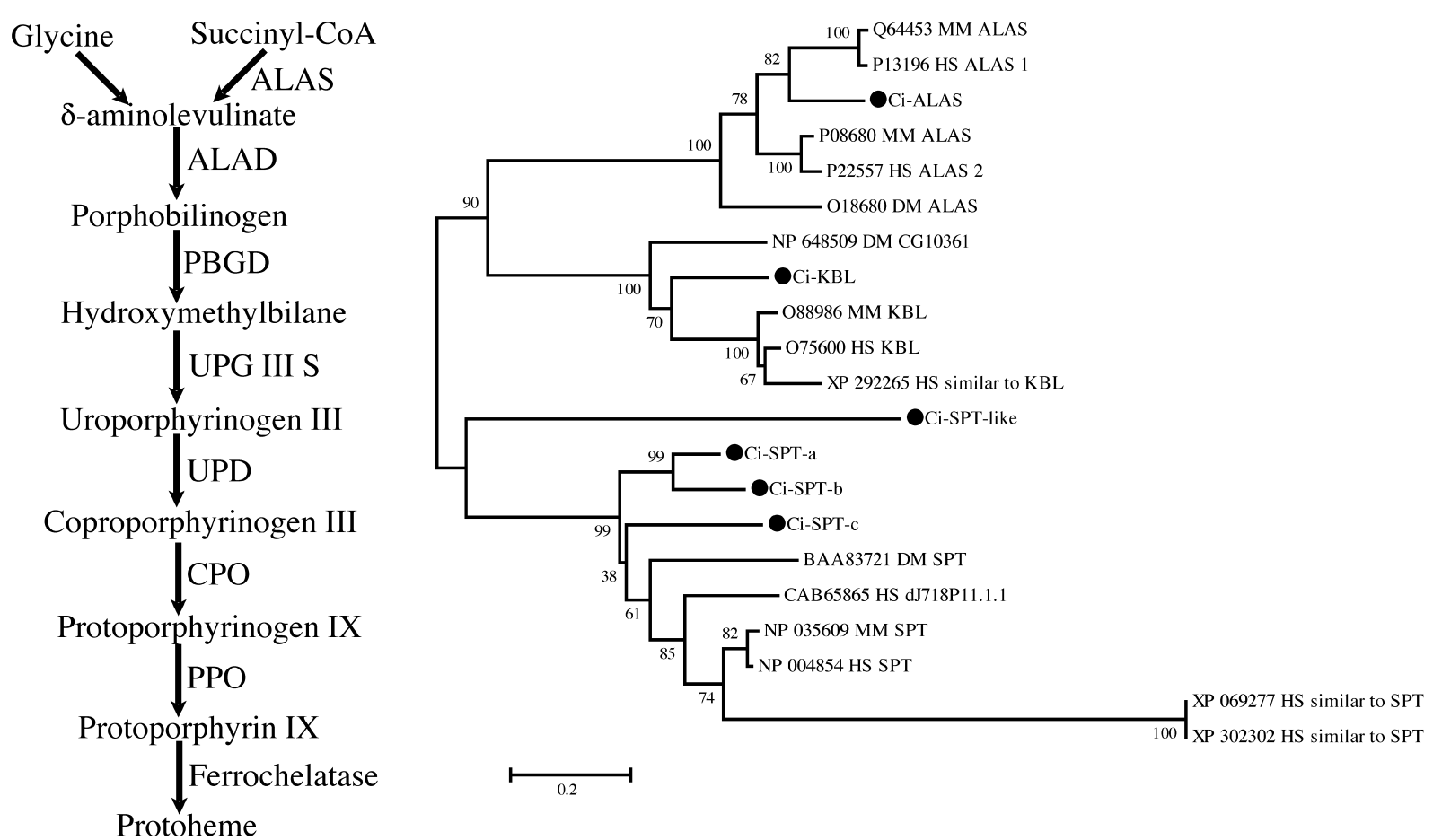

Fig. 7. (A) Heme biosynthesis pathway (Ferreira, 1995) and (B) phylogenetic tree of enzymes used for heme synthesis. The phylogenetic tree of (B) $\delta$-aminolevulinate synthase (ALAS) was generated by the neighbor joining method. Ciona intestinalis proteins are shown by large black dots. The number beside each branch indicates the percentage of times that a node was supported in 1000 bootstrap pseudoreplications. Protein names are explained in the Methods section. The unrooted tree is shown as a rooted tree for simplicity. The scale bar indicates an evolutionary distance of 0.2 amino acid substitutions per position. ALAD, $\delta$-aminolevulinate dehydratase; CPO, coproporphyrinogen oxidase; KBL, 2-amino-3-ketobutyrate coenzyme A ligase; PBGD, porphobilinogen deaminase; PPO, protoporphyrinogen oxidase; SPT, serine palmitoyltransferase, long chain base subunit 2; UPD, uroporphyrinogen decarboxylase; and UPG III S, uroporphyrinogen III synthase. 
A

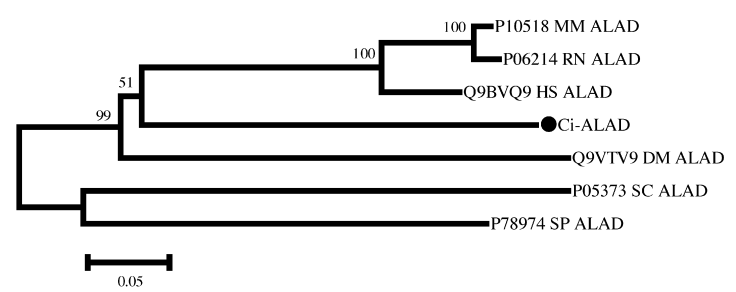

B

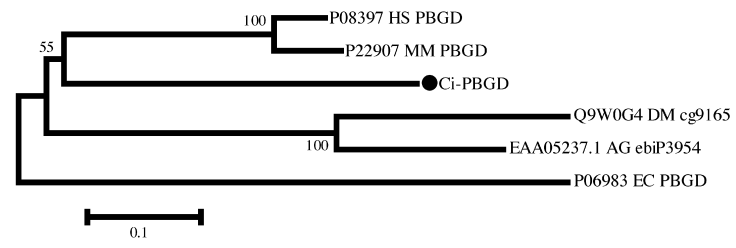

C

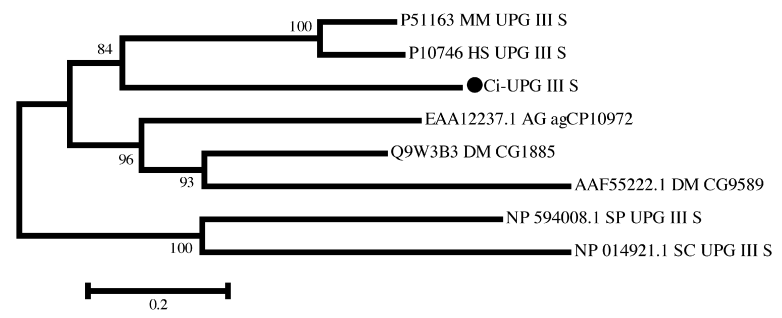

D

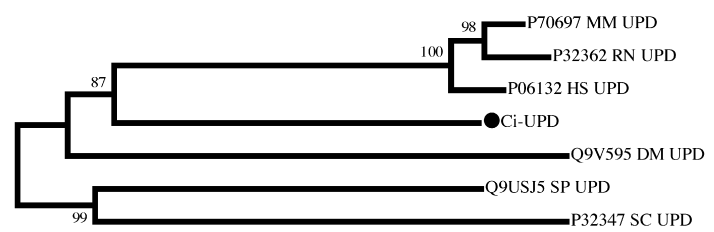

E
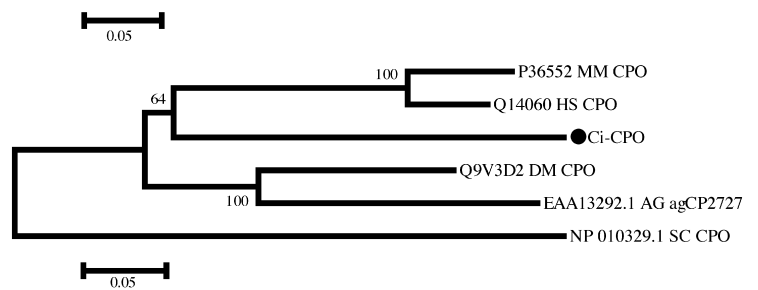

F

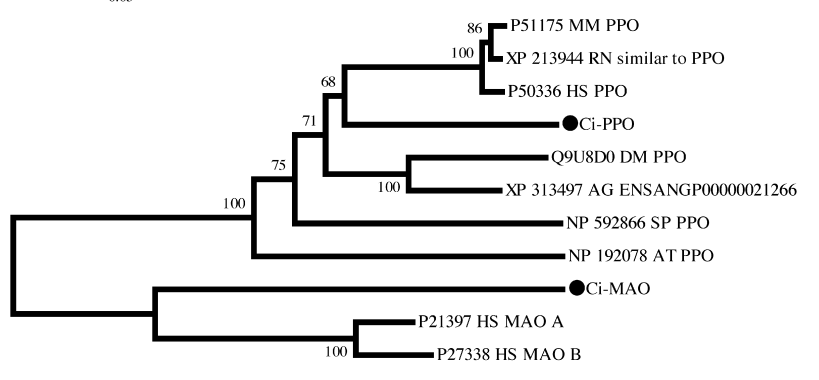

G

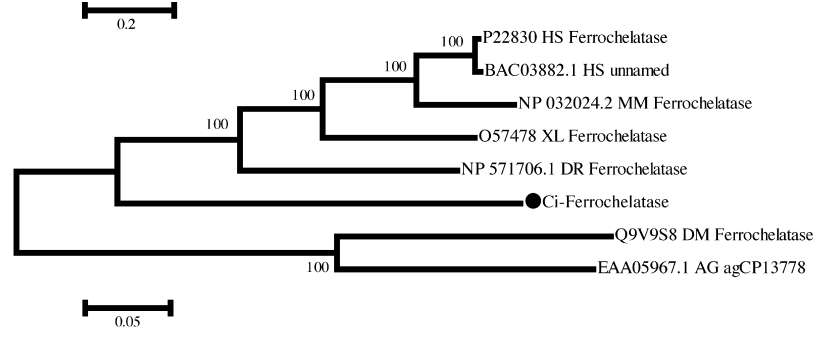

Fig. 8. Phylogenetic tree of enzymes used for heme synthesis. Phylogenetic trees of $(A) \delta$-aminolevulinate dehydratase (ALAD), (B) porphobilinogen deaminase (PBGD), (C) uroporphyrinogen III synthase (UPG III S), (D) uroporphyrinogen decarboxylase (UPD), (E) coproporphyrinogen oxidase CPO, $(\mathrm{F})$ protoporphyrinogen oxidase (PPO), and (G) ferrochelatase were generated by the neighbor joining method. Ciona intestinalis proteins are shown by large black dots. The number beside each branch indicates the percentage of times that a node was supported in 1000 bootstrap pseudoreplications. Protein names are explained in the Methods section. Each unrooted tree is shown as a rooted tree for simplicity. The scale bar indicates an evolutionary distance of 0.05 (A, D, E and G), 0.1 (B) or 0.2 (C, F) amino acid substitutions per position. 
nine. NBAD is transformed by phenoloxidase (PO) to NBAD quinone methide, from which papiliochrome and kynurenine are formed through non-enzymatic processes (Sugumaran et al., 1990). Kynurenine is an intermediate product of the ommochrome synthesis pathway, as mentioned above. On the other hand,NBAD is synthesized from tyrosine through dopamine. This pathway is partially shared with the melanin synthesis pathway and requires the enzymatic activity of dopa decarboxylase (DDC, EC 4.1.1.28). Synthesis of NBAD requires NBAD synthase, but the gene for this enzyme has not been fully sequenced.

A genome-wide survey of Ciona intestinalis genes for the enzymes in this pathway found two dopa decarboxylase (DDC)/histidine decarboxylase (HDC)-like genes (Fig. 6B). Molecular phylogenetic analysis indicated the duplication of this gene in the lineage leading to ascidians. However, the analysis did not provide evidence about whether these are true DDC or histidine decarboxylase genes (HDC, EC 4.1.1.22), or genes having other functions. Drosophila has both DDC and HDC, and thus the Ciona DDC/HDC-like genes do not appear to be ancestral forms of DDC and HDC. Therefore, it is not certain whether papiliochrome is synthesized in Ciona.

\section{Heme synthesis pathway}

Heme is not directly associated with pigment pattern, but many pigment proteins contain heme, and are thus associated indirectly with animal color patterns. The heme synthesis pathway and enzymes involved in the pathway are summarized in Fig. 7A. Components and enzymes of this pathway have been well conserved in prokaryotes and eukaryotes (reviewed by Ferreira, 1995). The heme synthesis pathway requires eight enzymes, starting from $\delta$-aminolevulinate synthase (ALAS, EC 2.3.1.37) which catalyses the first step of the pathway and thus acts as a rate-limiting factor. Heme is synthesized as the final product of the sequence of steps carried out by each of the eight enzymes.

Search of the Ciona intestinalis genome revealed eight genes that encode $\delta$-aminolevulinate synthase (ALAS) (Fig. 7B), $\delta$-aminolevulinate dehydratase (ALAD, EC 4.2.1.24) (Fig. 8A), porphobilinogen deaminase (PBGD, EC 2.5.1.61) (Fig. 8B), uroporphyrinogen-III synthase (UPG III S, EC 4.2.1.75) (Fig. 8C), uroporphyrinogen decarboxylase (UPD, EC 4.1.1.37) (Fig. 8D), coproporphyrinogen oxidase (CPO, EC 1.3.3.3) (Fig. 8E), protoprophyrinogen oxidase (PPO, EC 1.3.3.4) (Fig. 8F), and ferrochelatase (EC 4.99.1.1) (Fig. $8 G)$. Thus, Ciona has all the components for the heme synthesis pathway.

\section{CONCLUSIONS}

Our study revealed that the Ciona intestinalis genome contains genes for each enzyme of the melanin, pteridine, ommochrome, papiliochrome, and heme synthesis pathways. This is basic information for future studies of molecular mechanisms underlying the pigmentation of ascidians.
The present results are also useful to compare the relevant homologous genes between Ciona intestinalis and Ciona savignyi to investigate the presence or absence of red pigment spots in the rim of the sperm duct, a diagnostic character that distinguishes between the two species.

\section{ACKNOWLEDGMENTS}

This research was supported by a Grant-in-Aid for Scientific Research from MEXT, Japan to N.S. (12202001) and 21 COE (A14) for the Biodiversity Research.

\section{REFERENCES}

Allegri G, Costa CV, Bertazzo A, Biasiolo M, Ragazzi E (2003) Enzyme activities of tryptophan metabolism along the kynurenine pathway in various species of animals. Farmaco 58: 829836

Altschul SF, Madden TL, Schaffer AA, Zhang J, Zhang Z, Miller W, Lipman DJ (1997) Gapped BLAST and PSI-BLAST: a new generation of protein database search programs. Nucleic Acids Res 25: 3389-3402

Bateman A, Birney E, Cerruti L, Durbin R, Etwiller L, Eddy SR, Griffiths-Jones S, Howe KL, Marshall M, Sonnhammer EL (2002) The Pfam protein families database. Nucleic Acids Res 30: 276-280

Burmester T (2002) Origin and evolution of arthropod hemocyanins and related proteins. J Comp Physiol [B] 172: 95-107

Dehal P, Satou Y, Campbell RK et al. (2002) The draft genome of Ciona intestinalis: insights into chordate and vertebrate origins. Science 298: 2157-2167

del Marmol V, Beermann F (1996) Tyrosinase and related proteins in mammalian pigmentation. FEBS Lett 381: 165-168

Felsenstein J (1993) Phylogeny Inference Package (PHYLIP). University of Washington, Seattle

Ferreira GC (1995) Heme biosynthesis: biochemistry, molecular biology, and relationship to disease. J Bioenerg Biomembr 27: $147-150$

García-Borrón JC, Solano F (2002) Molecular anatomy of tyrosinase and its related proteins: beyond the histidine-bound metal catalytic center. Pigment Cell Res 15: 162-173

Giordano E, Peluso I, Rendina R, Digilio A, Furia M (2003) The clot gene of Drosophila melanogaster encodes a conserved member of the thioredoxin-like protein superfamily. Mol Genet Genomics 268: 692-697

Han Q, Calvo E, Marinotti O, Fang J, Rizzi M, James AA, Li J (2003) Analysis of the wild-type and mutant genes encoding the enzyme kynurenine monooxygenase of the yellow fever mosquito, Aedes aegypti. Insect Mol Biol 12: 483-490

Hearing VJ, Tsukamoto K (1991) Enzymatic control of pigmentation in mammals. FASEB J 5: 2902-2909

Higgins DG, Sharp PM (1988) CLUSTAL: a package for performing multiple sequence alignment on a microcomputer. Gene 73: 237-244

Hoshino Z, Tokioka T (1967) An unusually robust Ciona from the northeastern coast of Honsyu Island, Japan. Publ Seto Mar Biol Lab 15: 275-290

Immesberger A, Burmester T (2004) Putative phenoloxidases in the tunicate Ciona intestinalis and the origin of the arthropod hemocyanin superfamily. J Comp Physiol [B] 174: 169-180

Koch PB, Behnecke B, Weigmann-Lenz M, Ffrench-Constant RH (2000) Insect pigmentation: activities of beta-alanyldopamine synthase in wing color patterns of wild-type and melanic mutant swallowtail butterfly Papilio glaucus. Pigment Cell Res 13 Suppl 


$$
\text { 8: } 54-58
$$

Kumar S, Tamura K, Jakobsen IB, Nei M (2001) MEGA2: molecular evolutionary genetics analysis software. Bioinformatics 17: 1244-1245

Lorenzen MD, Brown SJ, Denell RE, Beeman RW (2002) Cloning and characterization of the Tribolium castaneum eye-color genes encoding tryptophan oxygenase and kynurenine 3monooxygenase. Genetics 160: 225-234

Matsubara M., Tsusué M, Akino M (1963) Occurrence of two different enzymes in the silkworm, Bombyx mori, to reduce folate and sepiapterin. Nature 199: 908-909

Pabarcus MK, Casida JE (2002) Kynurenine formamidase: determination of primary structure and modeling-based prediction of tertiary structure and catalytic triad. Biochim Biophys Acta 1596: 201-211

Quigley IK, Parichy DM (2002) Pigment pattern formation in zebrafish: a model for developmental genetics and the evolution of form. Microsc Res Tech 58: 442-455

Reaume AG, Knecht DA, Chovnick A (1991) The rosy locus in Drosophila melanogaster: xanthine dehydrogenase and eye pigments. Genetics 129: 1099-1109

Saitou N, Nei M (1987) The neighbor-joining method: a new method for reconstructing phylogenetic trees. Mol Biol Evol 4: 406-425

Sato S, Masuya H, Numakunai T, Satoh N, Ikeo K, Gojobori T, Tamura K, Ide H, Takeuchi T, Yamamoto H (1997) Ascidian tyrosinase gene: its unique structure and expression in the developing brain. Dev Dyn 208: 363-374

Sato S, Toyoda R, Katsuyama Y, Saiga H, Numakunai T, Ikeo K, Gojobori T, Yajima I, Yamamoto H (1999) Structure and developmental expression of the ascidian TRP gene: insights into the evolution of pigment cell-specific gene expression. Dev Dyn 215: 225-237

Satou Y, Yamada L, Mochizuki Y, Takatori N, Kawashima T, Sasaki A, Hamaguchi M, Awazu S, Yagi K, Sasakura Y, Nakayama A, Ishikawa $\mathrm{H}$, Inaba K, Satoh N (2002) A cDNA resource from the basal chordate Ciona intestinalis. Genesis 33: 153-154

Satou Y, Imai KS, Levine M, Kohara Y, Rokhsar D, Satoh N (2003a) A genomewide survey of developmentally relevant genes in Ciona intestinalis. I. Genes for bHLH transcription factors. Dev Genes Evol 213: 213-221
Satou Y, Kawashima T, Kohara Y, Satoh N (2003b) Large scale EST analyses in Ciona intestinalis: Its application as Northern blot analyses. Dev Genes Evol 213: 314-318

Schultz J, Milpetz F, Bork P, Ponting CP (1998) SMART, a simple modular architecture research tool: identification of signaling domains. Proc Natl Acad Sci USA 95: 5857-5864

Searles LL, Voelker RA (1986) Molecular characterization of the Drosophila vermilion locus and its suppressible alleles. Proc Natl Acad Sci USA 83: 404-408

Sugumaran M, Saul SJ, Dali H (1990) On the mechanism of side chain oxidation of $\mathrm{N}$-beta-alanyldopamine by cuticular enzymes from Sarcophaga bullata. Arch Insect Biochem Physiol 15: 255-269

Sugumaran M (2002) Comparative biochemistry of eumelanogenesis and the protective roles of phenoloxidase and melanin in insects. Pigment Cell Res 15: 2-9

Tamura T (1983) Deficiency of xanthine dehydrogenase activity in the oq oily mutant of the silkworm, Bombyx mori. Jpn J Genet 58: $165-168$

Thony B, Auerbach G, Blau N (2000) Tetrahydrobiopterin biosynthesis, regeneration and functions. Biochem $\mathrm{J} 347: 1-16$

Warren WD, Palmer S, Howells AJ (1996) Molecular characterization of the cinnabar region of Drosophila melanogaster. identification of the cinnabar transcription unit. Genetica 98: 249-262

Wiederrecht GJ, Paton DR, Brown GM (1984) Enzymatic conversion of dihydroneopterin triphosphate to the pyrimidodiazepine intermediate involved in the biosynthesis of the drosopterins in Drosophila melanogaster. J Biol Chem 259: 2195-2200

Wiederrecht GJ, Brown GM (1984) Purification and properties of the enzymes from Drosophila melanogaster that catalyze the conversion of dihydroneopterin triphosphate to the pyrimidodiazepine precursor of the drosopterins. J Biol Chem 259: 1412114127

Ziegler I (2003) The pteridine pathway in zebrafish: regulation and specification during the determination of neural crest cell-fate. Pigment Cell Res 16: 172-182

(Received December 20, 2004 / Accepted May 15, 2005) 